\title{
Application of microencapsulated biopesticides to improve repellent finishing of cotton fabrics
}

\author{
Maria M. Miro Specos ${ }^{a}$, Juan J. Garciab ${ }^{b}$ Alejandra C. Gutierrez ${ }^{b}$ and Laura G. Hermidac \\ ${ }^{a}$ Centro de Investigación y Desarrollo en Textiles, Instituto Nacional de Tecnología Industrial (INTI), Buenos Aires, Argentina; bentro de Estudios \\ Parasitológicos y de Vectores (CEPAVE), Consejo Nacional de Investigaciones Científicas y Técnicas (CONICET), Buenos Aires, Argentina; ${ }^{\mathrm{C} C e n t r o}$ \\ Investigación y Desarrollo en Química, Instituto Nacional de Tecnología Industrial (INTI), Buenos Aires, Argentina
}

\begin{abstract}
Insect repellent textiles are currently being investigated as a complementary tool to control disease vectors, such as mosquitoes. The application of microencapsulated biopesticides to textiles is a promising low-toxicity alternative to other control methods, as microcapsules may prolong the release of the repellent agent. In this work, microcapsules containing two biopesticides, namely citronella essential oil and citriodiol ${ }^{\oplus}$ were prepared and applied to cotton textiles using a variety of techniques. The aim of this work was to evaluate these functional textiles to select the most efficient, durable systems. Citriodioltreated cotton presented a prolonged durability, and 100\% repellency could be achieved for more than 30 days after padding onto cotton fabrics. It has to be pointed out that all these repellent textiles have been obtained by scalable, low-cost methods which would require no additional investment for textile finishing industries.
\end{abstract}

\section{ARTICLE HISTORY}

Received 8 June 2015

Accepted 2 November 2016

\section{KEYWORDS}

Repellent textiles; citriodiol; citronella oil; microencapsulation; Aedes aegypti

\section{Introduction}

The functionalization of textile surfaces with microencapsulated functional agents has enabled the development of fibers and fabrics with improved and new properties, such as aromatic (Specos et al., 2010), thermoregulation (Nejman, Cieslak, Gajdzicki, Goetzendorf-Grabowska, \& Karaszewska, 2014), flame retardancy (Salaün, Giraud, Vroman, \& Rault, 2013), UV protection (Vílchez-Maldonado, Calderó, Esquena, \& Molina, 2014), antibacterial (Nithyakalyani, Ramachandran, \& Rajendran, 2012), photochromic effects (Feczkó, Samu, Wenzel, Neral, \& Voncina, 2013), or combinations of two or more properties in one fabric, namely multifunctional textiles (Kolte \& Sharma, 2014). Microencapsulation could be defined as the process of covering a substance with materials of different nature in order to obtain particles of micrometric size. Functional agents are therefore protected from environmental factors and their release can be controlled by diffusion through the capsule wall, pressure, and/ or rupture of the microcapsules. The homogeneous application of microcapsules on textile materials might enable an increase in the durability of a desired effect in versatile textile finishing.

Mosquitoes are insects of public health concern because many species are vectors of diseases. Aedes aegypti is the primary vector of dengue fever, an important arboviral human infection worldwide. The urgent need for global actions to avoid further disease spread has led many researchers to evaluate different strategies for the control of mosquito bites. Various essential oils have been reported as mosquito repellents and promoted due to their low toxicity and eco-friendly nature (Amer \& Mehlhorn, 2006; Gillij, Gleiser, \& Zygadlo, 2008; Sritabutra \& Soonwera, 2003). Among them, citronella oil has been found to possess effective repellent activity against $\mathrm{A}$. aegypti females for $2 \mathrm{~h}$ after the direct application of undiluted oil on the skin of human subjects (Trongtokit, Rongsriyam, Komalamisra, \& Apiwathnasorn, 2005). Citriodiol ${ }^{\circ}$ is one of the WHO recommended biopesticides for use on skin and clothing. In fact, it has been tested and registered by the United States Environmental Protection Agency (EPA) and included in the European Biocidal Products Directive (BPD) 98/8/EC. It is a naturally derived mixture of substances which contains at least $64 \%$ of both cis and trans isomers of pmenthane-3,8-diol (PMD) and can be obtained from Eucalyptus citriodiora oil as previously described (Drapeau et al., 2011; Yuasa, Tsuruta, \& Yuasa, 2000). As a monoterpene, PMD has lower volatility than other plant derived compounds, resulting in a longer protection time (Barasa, Ndiege, Lwande, \& Hassanali, 2002). A formulation containing 20\% PMD concentration, for example, has been reported to provide protection against A. aegypti for more than 5 h (Carroll \& Loye, 2006).

The incorporation of natural insect repellents in textiles seems an alternative to provide longer lasting protection against mosquito bites while avoiding the contact with the skin, but their direct application to fabrics has proven to be inefficient to prolong 
the repellency long enough (Specos et al., 2010). Therefore, the use of microcapsules could provide a durable repellent finish that could be applied to a multitude of fibers (Li, Lewis, Stewart, Qian, \& Boyter, 2008).

Mosquito repellent fabrics have been previously developed by polymer coating methods (Faulde, Albiez, \& Nehring, 2012), by impregnation or surface-coating with cypermethrin (Hebeish, Hamdy, El-Sawy, \& Abdel-Mohdy, 2010), by encapsulation of DEET in chitosan microcapsules (Fei \& Xin, 2007), by inclusion of DEET, permethrin, or limonene in cyclodextrins grafted to textile substrates (Hebeish, Fouda, Hamdy, El-Sawy, \& AbdelMohdy, 2008; Romi, Lo Nostro, Bocci, Ridi, \& Baglioni, 2005), or by application of permethrin through a sol-gel treatment (Ardanuy, Faccini, Amantia, Aubouy, \& Borja, 2014). Textiles coated with synthetic pesticides using different technologies generally claim to be effective for a few months according to the specific application. However, studies on residual repellency of encapsulated natural repellents applied to textiles are limited to citronella microcapsules obtained by complex coacervation and applied on cotton fabrics, which provided 20 days protection against $\mathrm{A}$. aegypti mosquitoes (Specos et al., 2010).

The objective of this work is to obtain long lasting repellent textiles using different textile finishing strategies based on microencapsulated biopesticides. In this case, a naturally derived repellent, namely citriodiol, has been used as an alternative to citronella essential oil. Both repellents have been encapsulated by complex coacervation using gelatine and Arabic Gum as wall materials. Commercial melamine-formaldehyde microcapsules containing citriodiol have also been included to compare their performance to complex coacervation microcapsules and evaluate their potential washing resistance. Microencapsulated citriodiol and citronella were applied to cotton textiles using different finishing protocols. The repellency of treated textiles to A. aegypti mosquitoes was tested by an in vivo assay and the durability of the obtained fabrics was assessed. The controlled release microsystems have been selected due to the low-cost and the biocompatible nature of the ingredients involved, which is most desirable in products intended for human use. It is worth to mention that all the processes have been designed and performed in a textile pilot plant, so they could be scaled up to production.

\section{Materials and methods}

\section{Materials}

Bleached $100 \%$ cotton plain weaves with a specific weight of $140 \mathrm{~g} / \mathrm{m}^{2}$ were provided by Karatex S.A. (Buenos Aires, Argentina). Citriodiol ${ }^{\circ}$ from Citrefine International Ltd. (Leeds, U.K.) and citronella essential oil from Fornasari Ltd. (Buenos Aires, Argentina) were used as mosquito repellents. Type $125 \mathrm{~B}$ gelatin acquired from Parafarm (Buenos Aires, Argentina) and Arabic Gum acquired from Van Rossum y Cia. Ltd. (Buenos Aires, Argentina) were employed as wall materials and glutardialdehyde $25 \%$ w/w acquired from Merck \& Co. Inc. (Hohenbrunn, Germany) was used as hardening agent.

Melamine-formaldehyde microcapsules containing citriodiol (GEM-diol) were provided by Geminnov (France), who claimed that microcapsules slurry contained $50 \%$ microcapsules concentration with 4:1 oil to polymer ratio. Cationic fixer used for exhaustion bath application was a Tinofix FRD provided by Huntsman Argentina S.R.L. Solusoft UP, a non-ionic softener and Appretan 9202, an acrylic binder were both provided by Clariant Argentina and used for impregnation bath application. All chemicals were reagent-grade.

\section{Preparation and characterization of microcapsules}

Microencapsulation of citriodiol or citronella essential oil was performed by complex coacervation as previously described (Leclercq, Harlander, \& Reineccius, 2009). Briefly, an o/w emulsion was prepared by homogenization of $1.25 \% \mathrm{w} / \mathrm{v}$ gelatine, $0.85 \% \mathrm{w} / \mathrm{v}$ Arabic Gum, and repellent oil with 4:1 oil to wall materials ratio. $\mathrm{O} / \mathrm{W}$ emulsions containing gelatin, Arabic Gum, and citronella or citriodiol were thus obtained for textile treatment (EM-nella and EM-diol, respectively). Coacervation was achieved by dilution of gelatine-Arabic Gum emulsions with distilled water. The suspensions of gelatine-gum Arabic microcapsules containing citronella or citridodiol (GG-nella and GG-diol, respectively) were kept upon magnetic stirring overnight at room temperature. Microcapsules slurries were spray dried to evaluate their morphology in a Buchi Mini Spray Drier B290 (Flawil, Switzerland). Free-flowing powders were obtained and further analyzed by scanning electron microscopy (SEM) in a Philips SEM 505 (Philips/FEI, Eindhoven, Netherlands).

\section{Textile treatment and evaluation}

Fabrics were treated with emulsified or microencapsulated essential oils as follows. Cotton woven fabrics were padded in a Mathis scale foulard (Oberhasli, Switzerland) through an aqueous finish bath containing emulsified or microencapsulated oils, acrylic binder, and/or non-ionic softener. They were nipped to obtain a wet pickup of $100 \%$. Alternatively, fabrics were treated by bath exhaustion in a Mathis scale labomat (Oberhasli, Switzerland) with an acid bath containing microcapsules and a cationic fixer with a $20: 1$ liquor to goods ratio, at $40{ }^{\circ} \mathrm{C}$ for $30 \mathrm{~min}$. Samples were dried and cured when required in a Mathis scale tenter frame (Oberhasli, Switzerland). Drying and curing conditions were studied.

Laundering test was performed according to standard ISO 105-C06:2010(E) using a Gyrowash (James H Heal E Co. Ltd) for $35 \mathrm{~min}$ at $40^{\circ} \mathrm{C}$, avoiding the use of stainless steel balls. Samples were then rinsed with abundant water and air dried. Table 1 describes a series of experiments intended to improve repellency durability and/or washing resistance.

The remaining fragrance of treated samples was evaluated by three judges by smelling the swatch after scratching an ' $x$ ' on the specimen with a plastic stick. Samples were measured on a positive and negative scale about the presence of the fragrance (Li, Boyter, \& Qian, 2005).

\section{Test animals and repellency tests}

A. aegypti mosquitoes were reared in the laboratory using methods previously described (Gerber, Barnard, \& Ward, 1994). Adult mosquitoes were maintained in cages and fed with a $10 \%$ sucrose solution. Treated and non-treated fabrics were used to make 
Table 1. Finishing bath formula and treatment conditions for cotton or non-woven fabrics treated with emulsified or microencapsulated essential oils.

\begin{tabular}{|c|c|c|c|c|c|c|c|}
\hline \multirow[b]{2}{*}{ Component } & \multicolumn{5}{|c|}{ Foulard impregnation (A-E) } & \multicolumn{2}{|c|}{ Labomat exhaustion (F-G) } \\
\hline & Bath A & Bath $B^{b}$ & Bath C & Bath $D^{c}$ & Bath $\mathrm{E}^{\mathrm{d}}$ & Bath F & Bath G \\
\hline $\begin{array}{l}\text { Microcapsules concentration } \\
\text { (\%) }\end{array}$ & 10.4 & 5.2 & 4.6 & 1.5 & 2.1 & 1.4 & 5.2 \\
\hline Acrylic binder $45 \%$ solid (g/L) & & & 25 & 15 & 152 & & \\
\hline Non-ionic softener $(\mathrm{g} / \mathrm{L})$ & & & & & 13 & & \\
\hline Cationic fixer (\%)a & & & & & & 1 & 1 \\
\hline Liquor ratio & & & & & & $20: 1$ & $20: 1$ \\
\hline \multicolumn{8}{|l|}{ Treatment conditions } \\
\hline Pick up & 100 & 100 & 100 & 90 & 80 & & \\
\hline Single/double pad & double & double & double & single & single & & \\
\hline Drying temp. $\left({ }^{\circ} \mathrm{C}\right)$ & 80 & 80 & 120 & 120 & 100 & 80 & 80 \\
\hline Drying time (min) & 3 & 3 & 2 & 10 & 15 & 3 & 3 \\
\hline Curing temp. $\left({ }^{\circ} \mathrm{C}\right)$ & & & 150 & & 163 & & \\
\hline Curing time (min) & & & 1 & & 1 & & \\
\hline
\end{tabular}

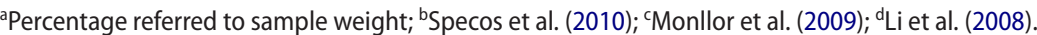

gloves to fully cover a hand and forearm. The repellent activity was evaluated by inserting the covered arm and forearm into a test chamber $(50 \times 50 \times 50 \mathrm{~cm})$, which was based on cage tests described in bibliography (Fei \& Xin, 2007). Test samples were kept for five minutes in the test chamber containing approximately 200 A. aegypti adult females which had not been fed for three to five days. The number of insects landing was counted independently by two observers. The trials were conducted in quintuplicate in five different cages at $26 \pm 1^{\circ} \mathrm{C}$ and $80 \pm 5 \% \mathrm{RH}$ with a 5 min waiting period between replicates. Samples were hung at room temperature in the open-air during the period of the assay.

The experiments were performed with the written consent of the participants according to the regulations of Bioethics and Biosecurity of the National University of La Plata and of the province of Buenos Aires, Argentina.

\section{Data analysis}

Repellency was indirectly calculated from the percentage of insects landing on treated fabrics using Equation (1):

$$
\text { Mean repellency }(\%)=\frac{\sum_{i=1}^{n}[100-(\mathrm{TF} / \mathrm{UF}) \times 100]}{n} \text {. }
$$

(a)

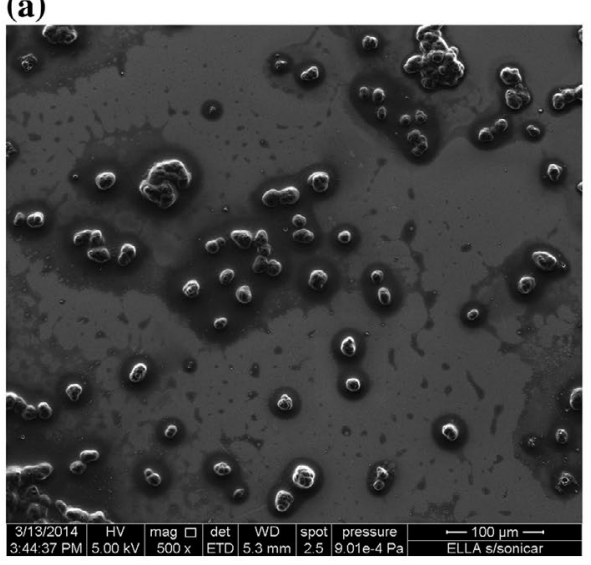

where TF and UF correspond to the number of mosquitoes landing on treated and untreated fabrics, respectively, and $n$ is the number of replicates for repellency assays, usually five.

\section{Results and discussion}

\section{Characterization of microcapsules}

Microcapsules containing citronella oil or citriodiol were characterized by SEM after spray drying. Microcapsules' size varied according to the encapsulated oil. In both cases, two types of structures can be observed: small spherical units of less than $10 \mu \mathrm{m}$ and agglomerates ranging from 25 to $100 \mu \mathrm{m}$. Citriodiol microcapsules (Figure 1(b)) were smaller than citronella microcapsules (Figure 1(a)), although more agglomerates were found.

Both mean size and size distribution of particles have to be considered for textile application. It has been assessed by our group that stained fabrics are obtained after padding with highly heterogeneous microcapsule slurries (data not shown). The stains are not only undesired features of any surface treatment, but also provide heterogeneity in the finishing, which would imply a heterogeneous repellency along the fabric as well. Similar criteria must be applied regarding microcapsule concentration. Highly concentrated microcapsule slurries could produce stained fabrics, depending not only on the suspension characteristics but

(b)

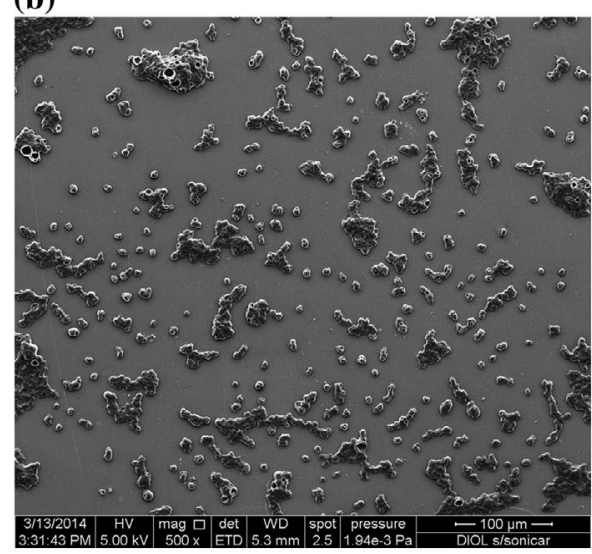

Figure 1. Scanning electron microphotographs of spray dried microcapsules containing (a) citronella essential oil and (b) Citriodiol; magnified x 500 . 
also on the textile material. Microcapsule agglomerates either in citronella or citriodiol suspensions were not profuse enough to stain the textile substrate in any case, and capsules were small enough to be well distributed among the fibers, allowing a fairly homogeneous functional finishing.

\section{Repellency of cotton fabrics padded with encapsulated or emulsified citronella oil or citriodiol}

The impregnation of textiles with microcapsule suspension without the use of any auxiliary reagent or curing protocol was carried out as a preliminary assay (Table 1, bath A). Figure 2(a) and (b) shows the surface appearance of fabrics treated with GG-nellabath A and GG-diol-bath A microcapsules, respectively, i.e. citronella and citriodiol in gelatin-Arabic Gum microcapsules applied to cotton in the conditions of bath A. Individual particles with a mean size around $20 \mu \mathrm{m}$ can be observed on cotton fibers, although some agglomerates are also noticed. The repellency durability of this first set of treated cotton gloves was evaluated as shown in Figure 3. It is clearly observed that both emulsified and encapsulated citriodiol (EM-diol-bath A and GG-diol-bath A) provided higher protection against mosquitoes than citronella formulations. Cotton fabrics treated with microencapsulated citronella presented more than $80 \%$ repellency for two weeks, while repellency for fabrics treated with microencapsulated citriodiol was higher than $95 \%$ for more than 30 days (Figure 3, white triangles). This is in agreement with the high repellent efficacy reported for citriodiol, which has been described as similar to that of synthetic repellents (Carroll \& Loye, 2006). It can be observed that citriodiol emulsion obtained with gelatin-Arabic Gum but avoiding coacervation, presented prolonged repellency durability, even higher than cotton treated with citronella microcapsules. This could be due to the formation of a thin film after padding with the polymer/repellent emulsion, which could act as a controlled release device after deposition onto fibers, as we had previously reported for citronella finishing (Specos et al., 2010).

Even when the polymers in the emulsion act as a barrier for citriodiol evaporation, resulting in good repellency, the microcapsule suspension has better stabibility than the oil emulsion. Microencapsulation is hence considered as an alternative for long-lasting repellency and stable formulations.

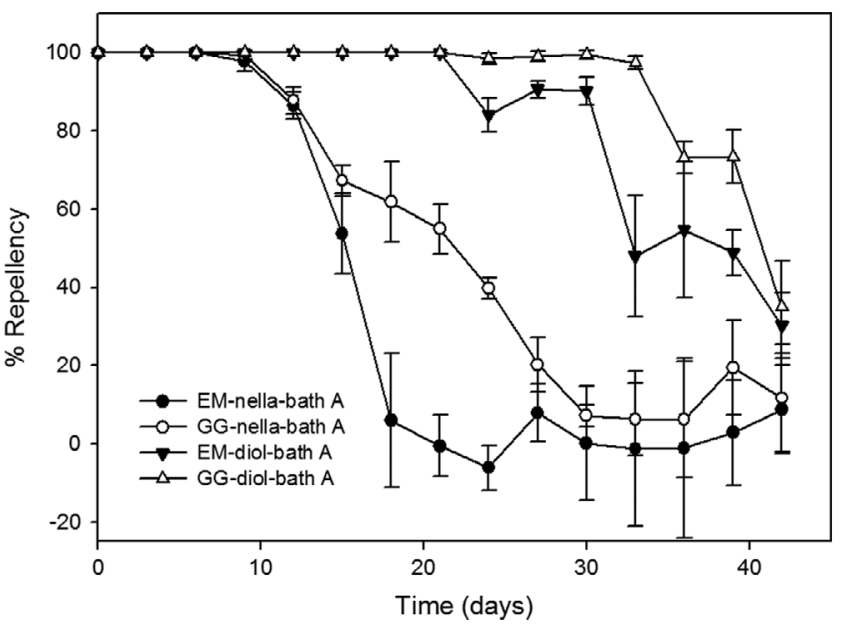

Figure 3. Variation of the repellency percentage as a function of time of cotton fabrics treated under bath $A$ conditions (Table 1), referred to untreated fabrics. Note: Bars show standard deviations for $n=5$.

\section{Effect of finishing methods on repellency durability and washing resistance}

Cotton fabrics were treated with citriodiol microcapsules by impregnation or exhaustion following different finishing protocols (Table 1). Commercial melamine-formaldehyde microcapsules containing citriodiol (GEM-diol) were included as they might show a higher resistance during application and further washing cycles. It must be noticed that bath B was similar to bath A, except for microcapsules concentration, which was diminished from $10.4 \%$ to $5.2 \%$ to optimize the cost/benefit ratio (Specos et al., 2010). In baths $C, D$, and $E$, an acrylic binder was used at different concentrations (Li et al., 2008; Monllor, Sánchez, Cases, \& Bonet, 2009), while baths $F$ and $G$ were applied by exhaustion and a cationic fixer was added to the finishing bath (Table 1). The use of binders and fixers is primarily to favor the fixation of the microcapsules on the textile surface and hence their washing fastness. Because microcapsules cannot react chemically with the fibers, binders could help the physical fixation by forming networks on the fabric. The selection of the acrylic binder and the cationic fixer was based on their common use in the industry. (a)

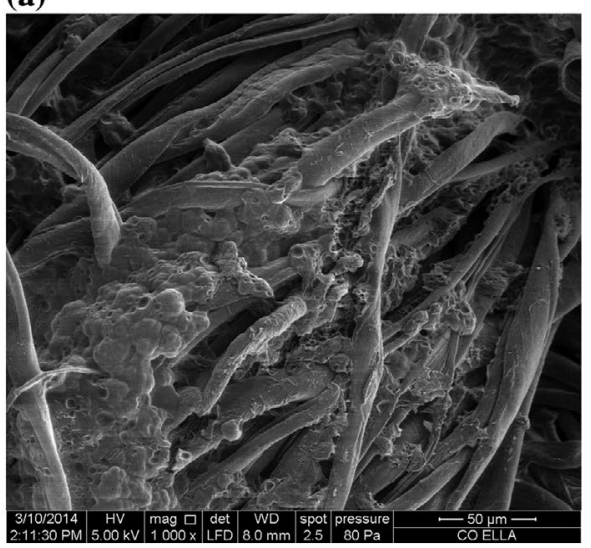

(b)

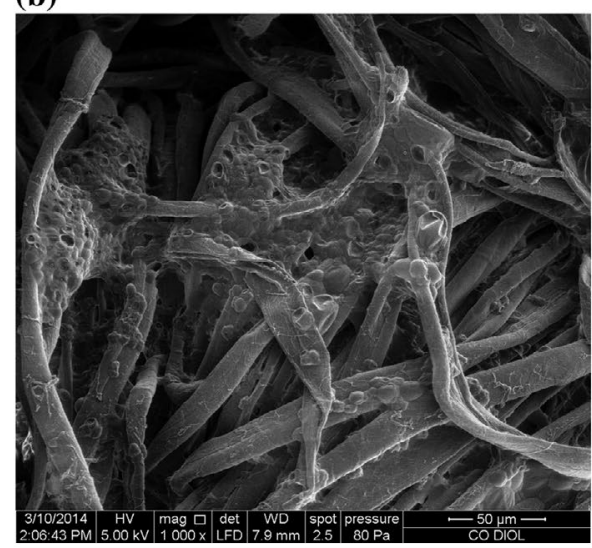

Figure 2. Scanning electron microphotographs of cotton fabrics treated under bath A conditions (Table 1) with (a) GG-nella microcapsules and (b) GG-diol microcapsules; magnified $x 1000$. 
(a)

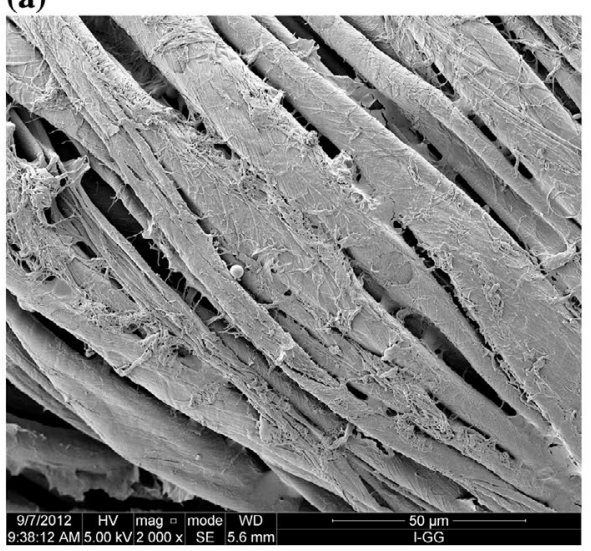

(b)

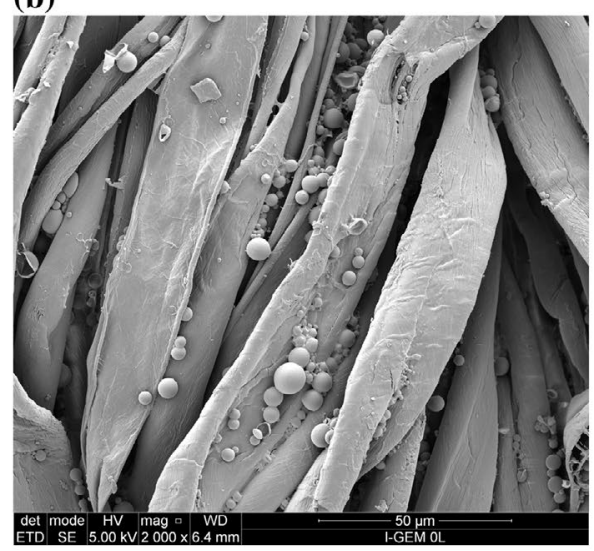

Figure 4. Scanning electron microphotographs of cotton textiles treated under bath B conditions (Table 1) with (a) GG-diol microcapsules and (b) GEM-diol microcapsules; magnified x 2000 .

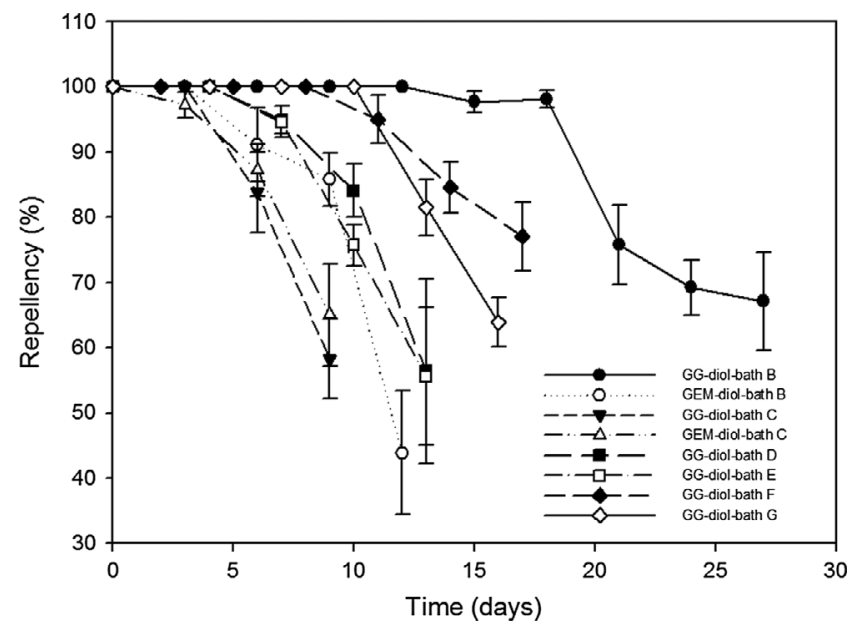

Figure 5. Variation of the repellency percentage as a function of time of cotton fabrics treated as described in Table 1, referred to untreated fabrics. Note: Bars show standard deviations for $n=5$.

Table 2. Olfactory evaluation of cotton fabrics treated as described in Table 1 with GG-diol or GEM-diol microcapsules.

\begin{tabular}{lcc}
\hline & \multicolumn{2}{c}{ Microcapsules } \\
\cline { 2 - 3 } Treatment conditions & GG-diol & GEM-diol \\
\hline Bath B & 1 & $>10$ \\
Bath C & 0 & $>10$ \\
Bath D & 0 & ND \\
Bath E & 0 & ND \\
Bath F & 8 & ND \\
Bath G & 2 & ND \\
\hline
\end{tabular}

Note: Numbers indicate the subsequent number of washing cycles after which the fragrance is still detected.

ND: non determined.

Figure 4 shows microphotographs of cotton fabrics padded with bath B containing GG-diol (Figure 4(a)) or GEM-diol (Figure 4(b)) microcapsules. Microstructures can hardly be observed on the fibers in GG-diol-bath B treated fabrics (Figure $4(\mathrm{a})$ ). A polymeric film is observed instead, probably due to the low mechanical strength of gelatine-Arabic Gum microcapsules, as it has been observed in a previous work (Specos et al., 2010). On the other hand, resin microcapsules (GEM-diol) show no changes in morphology after impregnation, the longest durability of the repellent effect was achieved through double padding of GG-diol without the use of auxiliaries or curing agents (Figure 5). Repellency was indeed $100 \%$ during 17 days and remained around $70 \%$ up to 28 days. The durability of the repellent effect was lower in this case compared to preliminary assays under bath A conditions with the same microcapsules (Figure 3), an expected fact given the dilution of the slurry. This is also in accordance with SEM observations, as a significant reduction in the amount of particles is also shown (Figure 2(b) vs. Figure 4(a)). No other treatment resulted in longer durability, in spite of the incorporation of auxiliary or curing agents (Figure 5, baths $\mathrm{C}-\mathrm{G})$. This finding is in accordance to our previous results showing that the use of auxiliary agents decreased the initial fragrance intensity on cotton treated with microencapsulated lemon essential oil (Specos et al., 2010). Particularly acrylic binders usually require high curing temperatures which favor the early release of volatiles. Moreover, they form a surface layer which prevents release.

Comparing baths $\mathrm{C}-\mathrm{E}$, it is observed that even when bath $\mathrm{C}$ has the highest concentration of microcapsules (4.6\%), the repellency of GG-diol-bath C-treated fabrics was very poor, less than five days and it significantly dropped afterward (Figure 5). While $\mathrm{D}$ and $\mathrm{E}$ have different microcapsules concentration (1.5\% y $2.1 \%$, respectively), repellency results are not significantly different between them but are better than GG-diol-bath C-treated fabrics. It can be inferred that neither the initial concentration of microcapsules padded nor the acrylic concentration in the treatment bath are determining effects to improve the repellency durability.

Olfactory evaluation is a complementary tool to characterize fabrics treated with microencapsulated volatile compounds. Repellency is actually an 'olfactory effect,' mainly dependent on the quick evaporation of the active compounds (Romi et al., 2005). In this case, it was used to assess the persistence of odor after 10 washing cycles as a preliminary assay before in vivo testing (Table 2). GG-diol microcapsules showed the lowest resistance to washing and only samples treated in bath $\mathrm{F}$ conditions could be smelt after eight washing cycles. 
Considering baths $\mathrm{F}$ and $\mathrm{G}$, is should be noted that bath $\mathrm{F}$ had almost four times less initial concentration of microcapsules than bath G. Still, GG-diol-bath F-treated fabrics presented higher washing resistance (Table 2) and longer repellent effect (Figure 5) than GG-diol-bath G-treated fabrics. These results are contrary to those obtained for GG-diol-bath A and GG-diolbath B-treated fabrics therefore suggest that the initial concentration of microcapsules padded is not the only parameter that affects repellency durability. These systems were not more deeply studied for this work, because none of them accomplished the expected repellency.

On the other hand, GEM-diol microcapsules presented odor even after 10 washing cycles for the assayed conditions, which could be promising for the development of washing-resistant finishings. It has to be considered however, that the application of these microcapsules to cotton in bath $\mathrm{B}$ conditions provided limited repellency durability, namely around ten days over $80 \%$ repellency (Figure 5). Citriodiol components which are responsible for the repellent effect may evaporate more easily than those responsible for a durable odor. Upon studying complex substances such as citriodiol, the presence or intensity of the odor might not be sufficient proof of repellency, given that individual components have different release profiles.

Regarding washing durability, microcapsules obtained by interfacial polymerization, such as those composed of mela$\mathrm{min}$ /urea-formaldehyde, have shown to present a relatively high mechanical strength (Hu, Chen, \& Zhang, 2009). However, volatile substances are prone to gradually release the active upon laundry processes even though they are encapsulated in resin reservoirs (Hebeish et al., 2008). Therefore, washing durability is limited to a few cleaning cycles even using dry washing, which is not the most common scenario (Rodrigues et al., 2009). Further assays will determine if odor evaluation for GEM-diolbath B samples correlates with prolonged repellency after washing which would be indicative of the washing resistance of the functional finishing.

It can be concluded that cotton fabrics treated with microencapsulated citriodiol can provide a prolonged repellency durability, namely $100 \%$ repellency for more than 30 days after treatment. The use of auxiliaries or curing agents neither improved the repellent effect nor its durability. Not even a high initial concentration of microcapsules is determinant to improve the repellent effect. It is stressed that the presence of odor in the fabric does not guarantee an effective repellency. It should be pointed out that the methodology described for achieving this kind of repellent fabrics is simple, low-cost, and reproducible, and requires no additional investments for textile finishing industries, a desirable factor in developing countries.

\section{Acknowledgments}

The authors would like to thank Miss Daiana Topollán for technical assistance in textile characterization and application and Mr. Juan Arata for assistance in the preparation of microcapsules.

\section{Disclosure statement}

No potential conflict of interest was reported by the authors.

\section{Funding}

This work was supported by the National Agency for the Promotion of Science and Technology (ANPCyT) through the FONARSEC program, Project FS Nano 002 Nanotechnology for Functional Textiles.

\section{References}

Amer, A., \& Mehlhorn, H. (2006). Repellency effect of forty-one essential oils against Aedes, Anopheles, and Culex mosquitoes. Parasitology Research, 99, 478-490.

Ardanuy, M., Faccini, M., Amantia, D., Aubouy, L., \& Borja, G. (2014). Preparation of durable insecticide cotton fabrics through sol-gel treatment with permethrin. Surface and Coatings Technology, 239, 132-137.

Barasa, S. S., Ndiege, I. O., Lwande, W., \& Hassanali, A. (2002). Repellent activities of stereoisomers of p-menthane-3,8-diols against anopheles gambiae (Diptera: Culicidae). Journal of Medical Entomology, 39, 736741.

Carroll, S. P., \& Loye, J. (2006). PMD, a registered botanical mosquito repellent with deet-like efficacy. Journal of the American Mosquito Control Association, 22, 507-514.

Drapeau, J., Rossano, M., Touraud, D., Obermayr, U., Geier, M., Rose, A., \& Kunz, W. (2011). Green synthesis of para-Menthane-3,8-diol from Eucalyptus citriodora: Application for repellent products. Comptes Rendus Chimie, 14, 629-635.

Faulde, M., Albiez, G., \& Nehring, O. (2012). Novel long-lasting impregnation technique transferred from clothing to bednets: Extended efficacy and residual activity of different pyrethroids against Aedes aegypti as shown by EN ISO 6330-standardized machine laundering. Parasitology Research, 110, 2341-2350.

Feczkó, T., Samu, K., Wenzel, K., Neral, B., \& Voncina, B. (2013). Textiles screen-printed with photochromic ethyl cellulose-spirooxazine composite nanoparticles. Coloration Technology, 129, 18-23.

Fei, B., \& Xin, J. H. (2007). N, N-diethyl-m-toluamide-containing microcapsules for bio-cloth finishing. American Journal of Tropical Medicine and Hygiene, 77, 52-57.

Gerber, F. J., Barnard, D. R., \& Ward, R. A. (1994). Manual for mosquito rearing and experimental techniques. American Mosquito Control Association Bull., 5, 1-98.

Gillij, Y. G., Gleiser, R. M., \& Zygadlo, J. A. (2008). Mosquito repellent activity of essential oils of aromatic plants growing in Argentina. Bioresource Technology, 99, 2507-2515.

Hebeish, A., Fouda, M. M. G., Hamdy, I. A., El-Sawy, S. M., \& AbdelMohdy, F. A. (2008). Preparation of durable insect repellent cotton fabric: Limonene as insecticide. Carbohydrate Polymers, 74, 268-273.

Hebeish, A., Hamdy, I. A., El-Sawy, S. M., \& Abdel-Mohdy, F. A. (2010). Preparation of durable insect repellent cotton fabric through treatment with a finishing formulation containing cypermethrin. The Journal of The Textile Institute, 101, 627-634

Hu, J., Chen, H. Q., \& Zhang, Z. (2009). Mechanical properties of melamine formaldehyde microcapsules for self-healing materials. Materials Chemistry and Physics, 118, 63-70.

Kolte, P. P., \& Sharma, A. S. (2014). Multifunctional finishing by a microencapsulation technique. International Dyer, 199, 23-27.

Leclercq, S., Harlander, K. R., \& Reineccius, G. A. (2009). Formation and characterization of microcapsules by complex coacervation with liquid or solid aroma cores. Flavour and Fragrance Journal, 24, 17-24.

Li, S., Boyter, H., \& Qian, L. (2005). UV curing for encapsulated aroma finish on cotton. The Journal of The Textile Institute, 96, 407-411.

Li, S., Lewis, J., Stewart, N., Qian, L., \& Boyter, H. (2008). Effect of finishing methods on washing durability of microencapsulated aroma finishing. The Journal of The Textile Institute, 99, 177-183.

Monllor, P., Sánchez, L., Cases, F., \& Bonet, M. A. (2009). Thermal behavior of microencapsulated fragrances on cotton fabrics. Textile Research Journal, 79, 365-380.

Nejman, A., Cieslak, M., Gajdzicki, B., Goetzendorf-Grabowska, B., \& Karaszewska, A. (2014). Methods of PCM microcapsules application and the thermal properties of modified knitted fabric. Thermochimica Acta, 589, 158-163. 
Nithyakalyani, D., Ramachandran, T., \& Rajendran, R. (2012). Microencapsulation of herbs for wound care textiles. Biosciences Biotechnology Research Asia, 9, 287-296.

Rodrigues, S. N., Martins, I. M., Fernandes, I. P., Gomes, P. B., Mata, V. G., Barreiro, M. F., \& Rodrigues, A. E. (2009). Scentfashion ${ }^{\circ}$ : Microencapsulated perfumes for textile application. Chemical Engineering Journal, 149, 463-472.

Romi, R., Lo Nostro, P., Bocci, E., Ridi, F., \& Baglioni, P. (2005). Bioengineering of a cellulosic fabric for insecticide delivery via grafted cyclodextrin. Biotechnology Progress, 21, 1724-1730.

Salaün, F., Giraud, S., Vroman, I., \& Rault, F. (2013). A review of microencapsulation of flame retardant formulations suitable for application in polypropylene textile substrates. In Polypropylene: Synthesis, applications and environmental concerns (pp. 195-222). New York, NY: Nova Science Publishers.

Specos, M. M. M., Escobar, G., Marino, P., Puggia, C., Tesoriero, M. V. D., \& Hermida, L. (2010). Aroma finishing of cotton fabrics by means of microencapsulation techniques. Journal of Industrial Textiles, 40, 13-32.
Specos, M. M. M., García, J. J., Tornesello, J., Marino, P., Vecchia, M. D., Tesoriero, M. V. D., \& Hermida, L. G. (2010). Microencapsulated citronella oil for mosquito repellent finishing of cotton textiles. Transactions of the Royal Society of Tropical Medicine and Hygiene, 104, 653-658.

Sritabutra, D., \& Soonwera, M. (2003). Repellent activity of herbal essential oils against Aedes aegypti (Linn.) and Culex quinquefasciatus (Say.). Asian Pacific Journal of Tropical Disease, 3, 271-276.

Trongtokit, Y., Rongsriyam, Y., Komalamisra, N., \& Apiwathnasorn, C. (2005). Comparative repellency of 38 essential oils against mosquito bites. Phytotherapy Research, 19, 303-309.

Vílchez-Maldonado, S., Calderó, G., Esquena, J., \& Molina, R. (2014). UV protective textiles by the deposition of functional ethylcellulose nanoparticles. Cellulose, 21, 2133-2145.

Yuasa, Y., Tsuruta, H., \& Yuasa, Y. (2000). A practical and efficient synthesis of p-Menthane-3,8-diols. Organic Process Research \& Development, 4, 159-161. 